\title{
FROM POINT CLOUD TO BIM: A MODELLING CHALLENGE IN THE CULTURAL HERITAGE FIELD
}

\author{
C. Tommasi ${ }^{* a}$, C. Achille ${ }^{\text {a }}$ F. Fassi ${ }^{\text {a }}$ \\ a Politecnico di Milano, 3D Survey Group, Dept. Of Architecture, Building Environment and Construction engineering, Via Ponzio \\ 31, 20133 Milan, Italy - (cinzia.tommasi, cristiana.achille, francesco.fassi)@polimi.it
}

\author{
Commission V, WG V/2
}

KEY WORDS: Real based model, Modelling, Cultural Heritage, BIM, Parametric modelling, Point cloud, Survey

\begin{abstract}
:
Speaking about modelling the Cultural Heritage, nowadays it is no longer enough to build the mute model of a monument, but it has to contain plenty of information inside it, especially when we refer to existing construction. For this reason, the aim of the research is to insert an historical building inside a BIM process, proposing in this way a working method that can build a reality based model and preserve the unicity of the elements. The question is: "What is the more useful mean in term of survey data management, level of detail, information and time savings?" To test the potentialities and the limits of this process we employed the most used software in the international market, taking as example some composed elements, made by regular and complex, but also modular parts. Once a final model is obtained, it is necessary to provide a test phase on the interoperability between the used software modules, in order to give a general picture of the state of art and to contribute to further studies on this subject.
\end{abstract}

\section{INTRODUCTION}

\subsection{Data management in the Cultural Heritage modelling}

The modelling process is different in an existing structure than in a new building: the target is to construct a real based model with a big scale of reference, generally 1:50, starting from the geometrical survey data and not from a project. The actual reduction of the time of surveying and acquisition of dense but rough three-dimensional measurements - point clouds - collides with modelling operations which are still largely manual and costly in terms of time-consuming and technology resources.

The point cloud is a direct data coming from both range based (laser scanner) and image based (photogrammetry) geometrical survey techniques, and it is the starting point for building the 3D model of a monument.

What makes difficult the managing of these data is:

1. The big size of the file;

2. The partial lack of interoperability between the survey tools and the modelling software;

3. The quite complicate phase of elaboration of the data.

If the representation needed is a $2 \mathrm{D}$ drawing (planimetry, section or elevation) the new functionalities offered by the software help the operator but are no longer enough for a complete description of the object. For this reason, the attention is focused on the creation of parametric and non-parametric models inside the BIM process, in order to be able to assign all the collected information to the historical building represented. Even if the management, elaboration and modelling phase of the point cloud is quite difficult, in the end it is possible to obtain satisfactory models. The aim of this paper is to present all the functionalities of the software used to elaborate this kind of data, both analyzing the general features of the programs and showing the process that brings the point cloud inside the BIM software, from the rough data to the model.

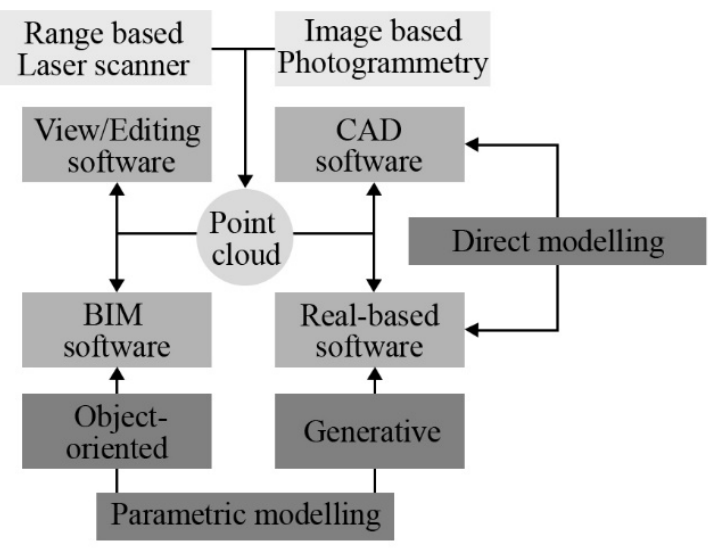

Figure 1. Point cloud management workflow: from the dense survey data to the different $3 \mathrm{D}$ modelling elaboration.

\subsection{State of art}

1.2.1 BIM experiences in $\mathbf{C H}$ field: During the last years the interest in using BIM in the Cultural Heritage field grew also because the potentiality of these kind of processes to help the users in the management and conservation of archaeological areas, monuments, artefacts, etc. Frequently, it is necessary to create systems ad hoc capable to bring together all the collected data, being sometimes more flexible and intuitive than the BIM software. Achille et al. (2015) wrote about this "Many European countries create informative platforms that try to collect all the data about their CH: e.g. the Kist o Riches by the School of Scottish Studies (University of Edinburgh, 2015), the CultureSampo developed by the Helsinki University of Technology (2015), and the Italian SICaR (2012). In particular, the Italian experience organizes the historical and scientific

\footnotetext{
${ }^{*}$ Corresponding author
} 
documentation in order to evaluate the interventions needed, the execution, the maintenance and the monitoring: this is a practical example of a system not only used for valorisation and tourism purposes, but also useful for restorers and operators of the construction yards."

In this regard, it is interesting to see how the BIM software have to be more and more shaped on the needs of Cultural Heritage, increasing the performances for the management of the data, overriding the gap between the competencies of one software or another and also being able to handle reality based models with information inside them.

An evolution is already in progress: it is enough to see how the features of the two most famous BIM software Autodesk Revit and Graphisoft Archicad, which were different and require the integration of plug-ins to be able to restitute an historical building starting from the point clouds (2013), now are perfectly aligned, and they don't need anymore external helps to correctly visualize and manage big data coming from the survey (2016).

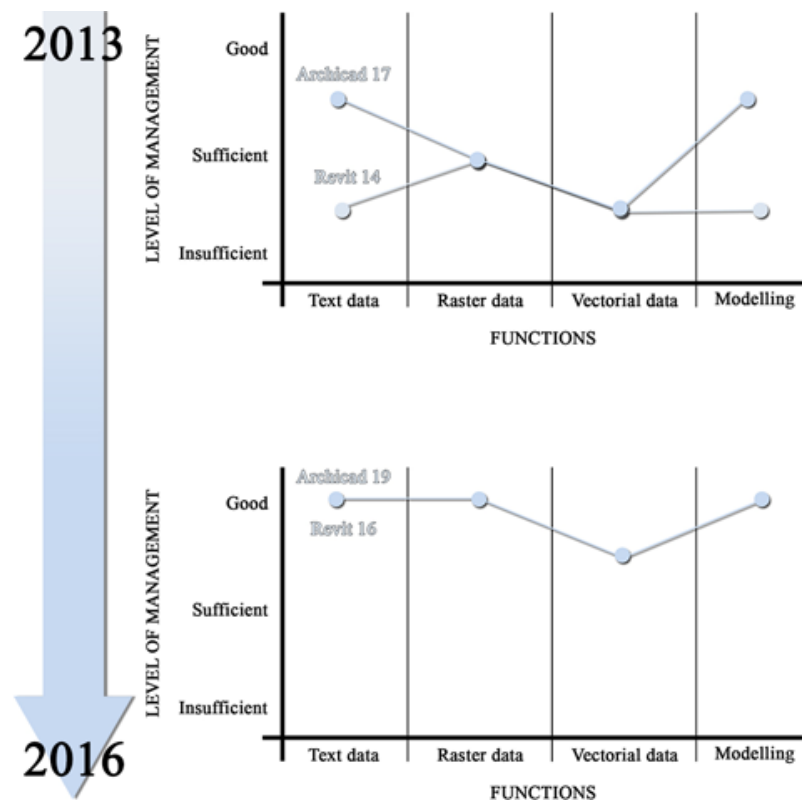

Figure 2. Evolution of the competencies and performances in data managing of two BIM software: Graphisoft Archicad and Autodesk Revit.

1.2.2 Software modules: Today, there are a lot of available software to work with point clouds that let their visualization, management and elaboration.

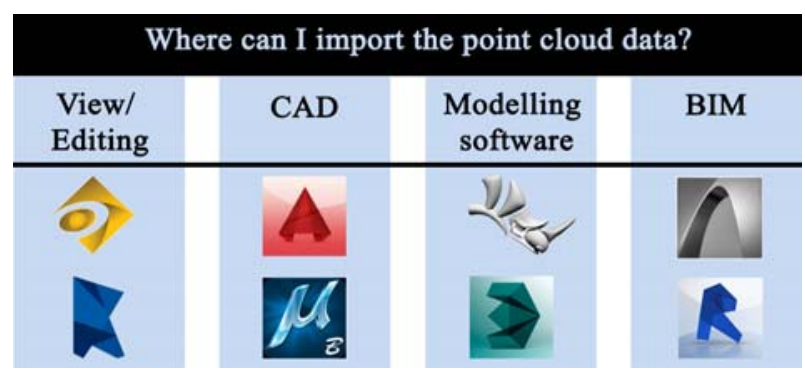

Figure 3. Categories and examples of software that import the point cloud data.

Essentially, it is possible to divide these programs in four categories:
1. View/editing software such as Bentley Pointools, Autodesk Recap, Pointfuse, Cloud Compare, Geomagic, Phidias, Descartes, Meshlab, Scalypso etc.

2. CAD software such as Autodesk Autocad and Bentley Microstation;

3. BIM software such as Autodesk Revit, Graphisoft Archicad and Allplan;

4. Modelling software as Rhino and 3Ds Studio Max;

The first category includes all the software that let to use the point cloud in the easier way: it is possible to view, explore, section and take linear measures directly from it, exporting both 3D, 2D and raster data.

Differently, the point cloud imported in CAD software can be used to extract 2D profiles and then create the 3D model, according to the traditional working method.

On the other hand, with the modelling software it is possible to generate directly the surface of the object and then extract profiles and sections. This technique, used in the Cultural Heritage field, elaborates the survey data and realizes 3D models characterized by high complexity, great accuracy, highresolution and heterogeneous features (both line based and surface based at the same time), in order to reconstruct the exact geometric shape of the original object.

Otherwise, the BIM process works in a complete different way, as it uses construction elements and object instead of mesh and nurbs, and most important, it is possible to add a lot of information to the part of the buildings, changing the traditional mute model and transforming it in a smart one. Only recently, these kind of software started to work with the point clouds, and using it to begin the 3D model makes the modelling part easier as it is possible to have at disposal all the measures and complex sections needed. Therefore, the advantages to use the BIM software to model the Cultural Heritage are not only in linking the information, but also in the modelling part. For this reason, it is important to underline the two main working method for building 3D models: the direct modelling and the parametric one.

\subsection{Direct modelling}

The direct modelling process starts with one of the software that import the point cloud and use it as base to vectorialize the profiles required for the $3 \mathrm{D}$ : from the data, it is possible to obtain the sections for the three-dimensional model. The modelling time in this approach mainly depends on the number of section to be vectored and on the complexity of the object. In fact, it is necessary to extract as many slices as the number of different profiles: shaping a complex object with this method, it is necessary a lot of time to realize an accurate model, because the need of a bigger number of horizontal and vertical sections.

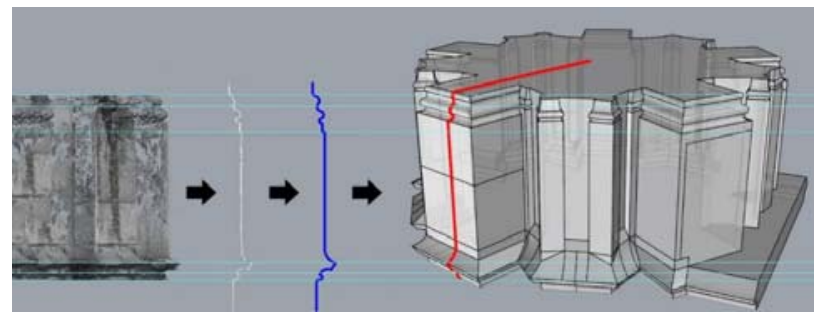

Figure 4. A "simple" geometrical object, where the vertical sections to be interpreted are at least ten.

The realization of 3D surfaces requires several passages and commands from the user and can result very tricky: for all these 
reasons, today the direct modelling is a "bottleneck" in the topographical survey field.

At the end of the workflow the element is unique, so if there are some similar parts in the building, hardly the model can be adapted to these. This is fitted on that surveyed data and for this reason, similar components require to begin the modelling from the first step.

\subsection{Parametric modelling}

The parametric modelling represents an alternative working method: this approach allows to work though pre-established models editable in any step of the process by the insertion of parameters. Nowadays this technique is used more in the realization of new buildings than in the restitution of the existing ones; however, it has great potential also in this second field. The two categories of parametric modelling are:

1. Parametric-generative models: they work with sequences of command and allow a very high level of freedom in modelling and consent to generate complex shapes (Grasshopper). It is mostly used in the new building field.

2. Parametric-object oriented models: this technique is based on the use of pre-built libraries and on the basic construction elements. The structure of these software makes them particularly suitable for being able to exploit the potential of BIM (Revit, ArchiCAD, VectorWorks).

As Tedeschi (2011) wrote, "The parametric software protagonist of a wide and trasversal spread thanks to an intuitive use that doesn't require programming knowledges - let to organize the projects in associative systems based on the relationships between parts, offering the possibility to alter the overall configuration of a system, by working on the parameters at the base of the design process, according to a logic of propagation of changes."

In this way the operator does not model from zero each element but, once modelled the first one, he can immediately extract the others, simply assigning the correct input data.

1.4.1 Parametric-generative modelling: The generative modelling requires a logical/mathematical approach because is based on the use of algorithms: for this reason, it is possible to speak also about algorithmic modelling, where, as Tedeschi (2011) wrote, "the goal achievement is always related to a proper detection and resolution of the different problems associated to a specific operative range. The resolving procedure is usually tied to the decomposition of the problem in a number of easier steps that ensure an efficient management of the process with traceability and transmissibility of the involved phases. This type of approach, combining cognitive mechanisms and human reasoning, is transferable to the pc with algorithms. In the informative technology field, the solution to a problem can be interpreted as a path through which you can get a result from initial data. These are defined input and the results are called output. The algorithm is the process for calculating a desired result from an input data. This is possible through a logical sequence of simple instructions, given by the operator and processed by the computer." The true goal is the definition of a general procedure for the solution of similar problems. This is probably the most relevant aspect, as it would create an abacus of algorithmic and parametric models that describes most of the similar element of a building. It could be a real and proper database composed by virtual elements that correspond to the shapes of the construction, and where every model corresponds to all the real elements with similar features but different dimensions.

Therefore, substantially, the generative modelling is applied in the construction of objects with similar shapes but different proportions and dimensions (typical elements of Cultural Heritage field) that if modelled with the direct method, each would require the realization of a model from zero, making the digital reconstruction phase onerous, time consuming and expensive. Realizing big restitution scale models, it is necessary to respect the diversity of the elements and consequently to follow their real shape: the expected result is the creation of a theoretic model, built on the primitive shapes and applicable to all the other elements of the same typology.

The phases of this working method are:

1. Deconstruction phase: to study the object and identify its generative shapes and the modalities in which it can be geometrically realized. The final operation of this step is the extraction of the profiles useful to the generation of the model's surface from the point cloud, which is the same operation of the direct modelling. The difference is that in this case, I have to do this operation only for one element. These profiles will be the input data of the generative modelling;

2. Reconstruction phase: creation of the parametric model, designing the command sequence. This is when the connections that elaborate the input data are created, giving as output the final model. The algorithmic relationship sets designed for the realization of the model are called clusters.

3. Concretization phase: transition between the parametric and the modelling environment (Rhinoceros);

One last step that could be included in concretization phase and that should be mandatory, is the test of accuracy: turning the model into a mesh, it is possible to overlap it over the point cloud and verify its fitting on the survey data. It is necessary that the difference between the model and the cloud is contented inside the tolerance fixed for the restitution scale given. That test is what distinguishes a reality based modelling from one not based on the reality.

1.4.2 Parametric object-oriented modelling: on the other hand, speaking about the object-oriented modelling, we refer essentially to the BIM working process, as they use standard constructive elements to build the model. Focalizing the attention on the word "standard" it is clear that these software are mostly used in new construction field, in fact, when we refer to the Cultural Heritage, we have to consider the unicity of the elements that build the monument. For this reason, only recently these programs have begun to develop more functionalities to handle the problems coming from the existing structures like the managing of the survey data (point cloud), the possibility to assign specific information to specific points of the element and the modelling of complex shapes. In the $\mathrm{CH}$ field, the starting point is not a project, but a geometrical survey and the BIM is inserted in the middle of the lifecycle of the building. Therefore, the first step is an architectural survey and then the goal is to handle all the collected information in a conservation project. For this reason, the general target of tests in progress is to investigate the possibility of using the parametric modelling, normally applied to new construction, to the existing ones. As told before, it happens in the existing building to find lots of similar elements, but with quite different dimensions (e.g. columns, arches, shingles, etc.): the ideal condition would be to model one element and repeat the others 
only changing the parameters and adjusting it on its personal shape and not starting over from the point cloud. This is not always possible, infact every object contained in these programs is parametric, and so editable in every part, but when it is modelled the Cultural heritage using complex elements and shapes, sometimes they are not modifiable. It is necessary to find the best way to speed up the process and obtain a high definition model.

\section{DUOMO CASE STUDY AND TESTS}

\subsection{The importance of a smart model in the Milan's cathedral}

For the research, we chose a very important and unique example in the international overview of the Cultural Heritage: The Milan's Cathedral. The reason of this choice is the need of constant maintenance of the church, that can be controlled by building a smart model with all the information about the decays and the restoring inside it. As Fassi et al. (2015) wrote about the maintenance of the Cathedral, "It is quite impossible to describe in few words the traditional methods to maintain the cathedral by Veneranda Fabbrica. Synthesizing at most, one can say that the main task of the Fabbrica is to guarantee the structural safety of the cathedral. [...] The Milan's Cathedral is a living monument and is experienced by people, it has to be safe and functional. It is built entirely in Candoglia marble that undergoes over the years strong structural weakening due to rain washout and especially to air pollution. [...] All the cathedral is monitored by careful periodic inspections, from which all maintenance activities are designated. Normally the main interventions operations consist in substitution, in whole or in part, of the degraded marble blocks, anchorages and reinforcement structures. Cleaning and restoration operations are normally scheduled for decorations and statuary. In this way the building site of the Milan Cathedral is an eternal construction site that regularly intervenes to heal those parts that threaten its stability and its security."

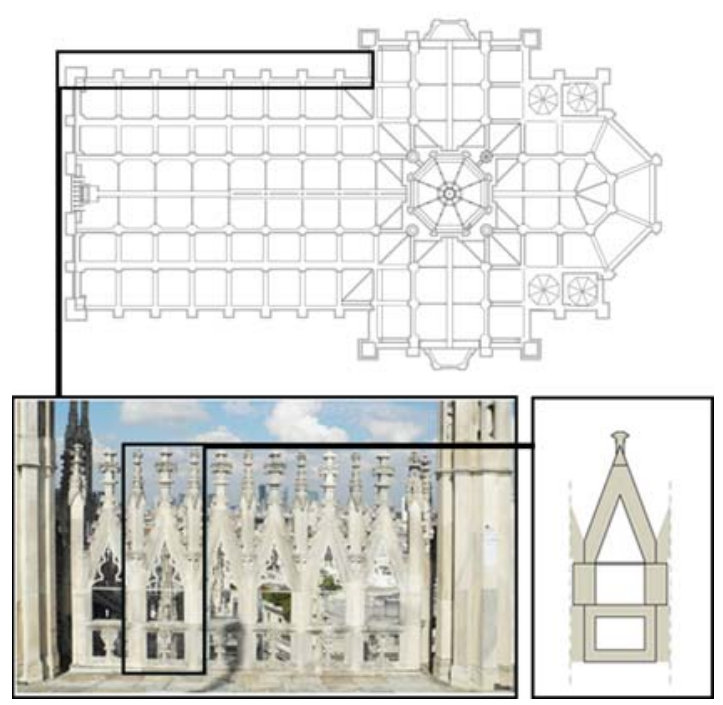

Figure 5. Localization and the composition of the element "Falconatura" on the rooftop plan of Milan's Cathedral.

2.2 The test

2.2.1 Shapes analysis - Deconstructive phase: To run some specific tests, we chose a part called "falconatura", which is modular and repeated in the entire cathedral, sometimes with same dimensions, and some other with different measures, made by regular and complex elements: this fact makes it the perfect object to be parameterized. Using the point cloud data from laser scanner and photogrammetry, integrated with direct measurements taken in situ, it was possible to understand that it is composed by constructive marble elements with the same profiles (e.g. as the pillars' or pinnacles' section), and the only dimensions that change is the interaxle space between the pillars that, changing time to time, modifies the angle between the pinnacles. Regarding the decorative parts, called "fiocco" and "gattone", they are unique and complex elements, to be modeled apart.

The target - Reconstructive phase: As told before, the starting data is a point cloud coming from laser scanner and photogrammetry and we elaborate it in different way: the direct modelling with Rhinoceros, the parametric - generative modelling with Grasshopper for Rhinoceros, and finally with the parametric object oriented modelling that belong to the BIM software, in this case Archicad 19 and Revit 16. As written before, the aim is to confront the different working method and find the best one that gives the best result for a real based model in 1:50 scale, and in the meantime, it helps monitoring the state of decay of the heritage with a system of information. For these reasons, it is clear that the creation of a parametric model divided in blocks, will let to i) speed up the modelling part and ii) assign to each of them the specific information, as the type of material, the decay, and the necessary or occurred substitution. Before arriving to the modelling part, it is necessary also to understand how the survey data are treated by the software, underlining the potentialities and the criticalities of managing big survey data in software born to be use in the new constructions field.

\subsection{Direct Modelling: Rhinoceros}

2.3.1 Import and visualization: Rhinoceros is a modelling software that has all the instruments and tools to obtain very high definition models. It can insert a *.txt or *.xyz point cloud, and it is able to visualize it well as a block and using it as tracing path, even if it doesn't recognize the points as snaps, and it is not possible to edit the cloud (cut it to create sections and profiles, etc.).

\begin{tabular}{|l|l|}
\hline Import format & \multicolumn{1}{|c|}{ xyz, txt, pod (Arena 4D) } \\
\hline $\begin{array}{l}\text { Import time (with a PC } \\
\text { intel i7, ram 16 GB and }\end{array}$ & 10 minutes with a cloud of 6 GB \\
\hline Visualization & \\
& $\begin{array}{l}\text { (Arena 4D) Possibility to have } \\
\text { different styles of visualization: } \\
\text { RGB, single color, normal, } \\
\text { intensity, altimetry }\end{array}$ \\
\hline Functions & $\begin{array}{l}\text { Retracing } \\
\text { Editing (Arena 4D) } \\
\text { Modelling }\end{array}$ \\
\hline
\end{tabular}

Table 1 . Summary of point cloud's import process in Rhino.

2.3.2 Modelling the cloud: Fortunately, to overcome these issues, and make the cloud modelling easier and more precise, there is a plug-in called Arena $4 \mathrm{D}$, which is the substitute of the old Pointools for Rhino, a useful tool to manage the point cloud, as it let to slice, clip, snap to point and run other editing 
function.

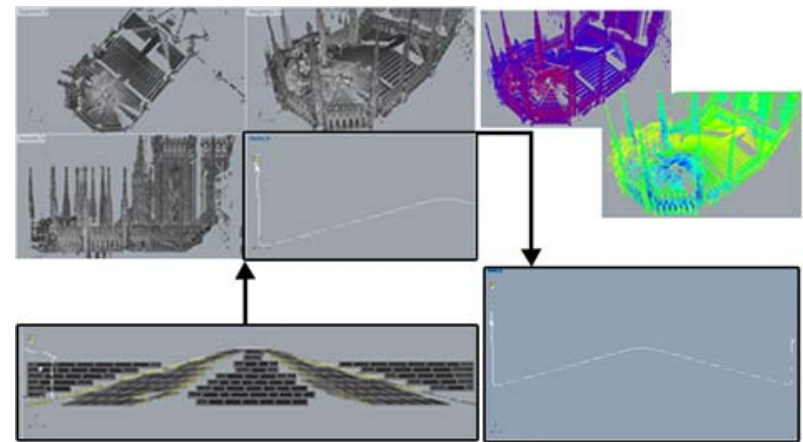

Figure 6. Importing and viewing the cloud. Top line: Importing a big size cloud with the possibility of different styles of visualization (on the right). Bottom line: cropped cloud, creation of profiles and sections.

In this way it is possible to achieve great detailed results, even if with two criticalities: one concerning the modelling part, and the other the information adding. Regarding the first one, as Rhino is not a parametric software itself (it can become one with the plug-in Grasshopper), it is not possible to modify the created objects assigning parameters to the various geometrical features belonging to the elements themselves (height, width, radius, etc.), but the editing is a result of actions external to them (move, rotate, stretch, etc.). It is clear that if there are a lot of similar objects with different dimensions, without the possibility to copy and adjust the geometrical features, theoretically the process is less immediate and more timeconsuming than a parametric software.

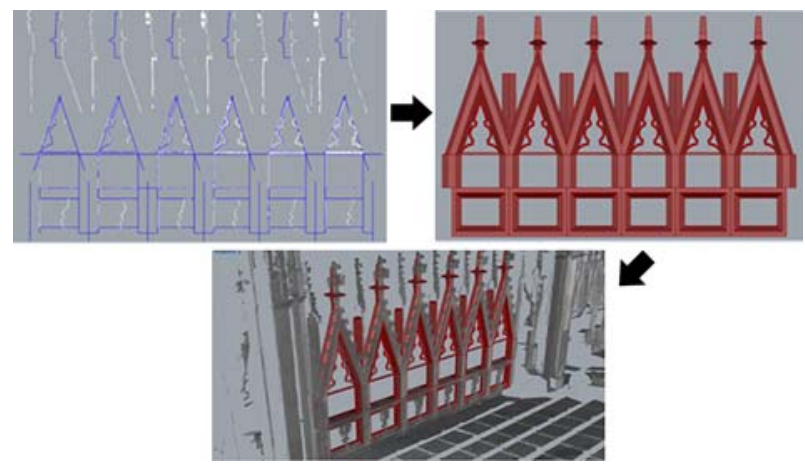

Figure 7. Starting from the point cloud, it was necessary to trace every section and model every single element, even if in this case the objects are similar, with only different dimensions: it was not possible to model one arch and adjust the others on the existing one.

Regarding the second issue, Rhinoceros does not consider the information adding, so the obtained model is mute, and for Cultural Heritage field's purposes useless as it can't help the restoring and the managing of the object or site. To overcome this critical need and in order to have both a high resolution model and a system of information linked to it, it is possible to find a research work by the 3D Survey Group from Politecnico di Milano.

\subsection{Parametric - generative modelling: Grasshopper}

2.4.1 Import and visualization: Grasshopper is a plug-in of
Rhino, so the import and the visualization of the survey data works in the same way,

2.4.2 Modelling the cloud: As told before, the generative modelling let to automate the procedures of the direct modelling, defining the shapes through mathematical functions, and consequentially, to generate parametrical models that allow quick and deep changes in the initial geometries, starting from the inputs of the survey data (point cloud). This type of modelling is based on algorithms, which are devices that permit to calculate the desired result starting from the input data. The crucial work is to interconnect the algorithms able to do automatically the modelling operations that lead to output of the final 3D model. The whole algorithms are a group of command projected by the operator, which are already present in Rhino (without Grasshopper) but this plug - in makes them also interactive. The result is to obtain a "cluster" between the input and the output data that represent the modelling part and which is always editable in every portion.

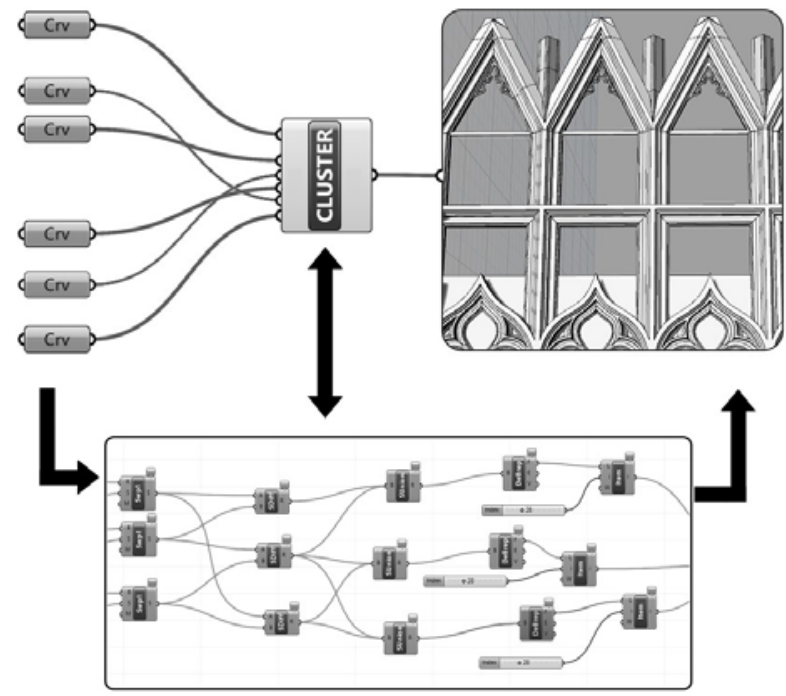

Figure 8. The workflow in Grasshopper to build a "falconatura". Obtaining the section of the elements (input $\mathrm{CRV}$ ) from the survey data and the final model (output) through a series of commands that together compose the cluster.

This workflow is theoretically irreproachable but presents a huge unsolved bug: sometimes, the connections between algorithms are not always successful, even if the cluster is well built. Particularly, the high complex clusters are not able to process determinate input data: this fact represent a problem in the Cultural Heritage field, where the most part of the elements are very articulate. When the whole process is investigated, the issue can be only one small algorithm that doesn't work (e.g. a boolean subtraction) and it is not possible to fix it. The result could be to not have a final output at all, or have it but wrong, and for this reason, it is unproposable to trust a system that randomly decides to not run, even if with great potentialities.

\subsection{Parametric object oriented modelling using the BIM process: Archicad 19}

2.5.1 Import and visualization: The test with Archicad 19 starts with the import of the complete point cloud of the Cathedral's rooftop. The file was opened in xyz and the importing time was $10^{\prime}$. The cloud did not have the RGB values and the object was dark because there where too much points. The visualization operations, as the zoom, was difficult in the 
point near the laser stations and easier in the parts more distant from the laser. To pass this first problem, the cloud was filtered and in Leica Cyclone: in this way, the file was light and there were and there were no more problems of visualization.
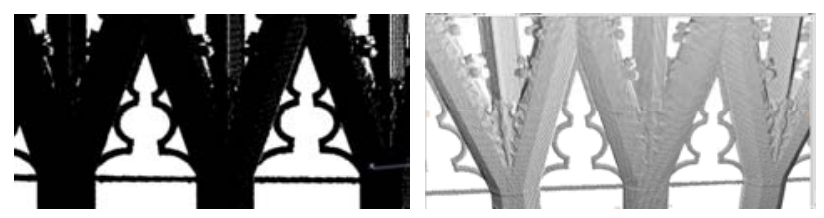

Figure 9. the visualization of the cloud before reducing it (on the left) and after (on the right).

The process of cloud managing was complex because it needed a pre-elaboration with other software, and it was necessary to filter the survey data to correctly view it. Anyway, once the file is optimized, the point cloud is processed without problems.

\begin{tabular}{|l|l|}
\hline Import format & \multicolumn{1}{|c|}{ xyz, e57 } \\
\hline $\begin{array}{l}\text { Import time (with a PC } \\
\text { intel i7, ram 16 GB } \\
\text { and 250 GB SSD) }\end{array}$ & $\begin{array}{l}\text { Almost immediate with a reduced } \\
\text { 1,5 GB size cloud }\end{array}$ \\
\hline Visualization & $\begin{array}{l}\text { With dense point cloud: } \\
\text { Zoom problems and so inability to } \\
\text { distinguish the points } \\
\text { If the pc is not powerful, the } \\
\text { viewing is slow }\end{array}$ \\
\hline Functions & $\begin{array}{l}\text { Retracing } \\
\text { Information adding }\end{array}$ \\
\hline
\end{tabular}

Table 3. Summary of point cloud's import process in Archicad.

2.5.2 Modelling the cloud: the modelling phase presented problems: in Archicad thus far, is not possible to draw complex profiles in elevation, and the point cloud is thought to only be used as tracing path or reference or to contain information, but not to be modelled with complex shapes. In conclusion, even if it is possible to draw primitives on the cloud, the modelling of complex sections still results difficult, as Archicad lack of $3 \mathrm{~d}$ modelling commands and even because it works in a completely different way than the traditional modeller (it is an object-oriented software).

\subsection{Parametric object oriented modelling using the BIM process: Revit 16}

2.6.1 Import and visualization: As other CAD software, before importing the point cloud data, Revit indexes it automatically, converting it in rcp format (Autodesk Recap). In this case the ptx file of the entire cloud was converted by the software, and once imported in the workspace, it appeared completely manageable, with different styles of visualization, snap points, and the possibility to section the cloud, see the created profiles and to see simultaneously more views and section.

\begin{tabular}{|l|c|}
\hline Import format & \multicolumn{1}{|c|}{ ptx, xyz, txt, rcp } \\
\hline Import time (with a PC & 1 hour with a cloud of 6 GB \\
intel i7, ram 16 GB & \\
and 250 GB SSD) & \\
\hline Visualization & \\
\hline
\end{tabular}

\begin{tabular}{|l|l|}
\hline & $\begin{array}{l}\text { Possibility to have different styles } \\
\text { of visualization: RGB, single } \\
\text { color, normal, intensity, altimetry. }\end{array}$ \\
\hline Functions & $\begin{array}{l}\text { Retracing } \\
\text { Editing } \\
\text { Modelling } \\
\text { Information adding }\end{array}$ \\
\hline
\end{tabular}

Table 4. Summary of point cloud's import process in Revit.

2.6.2 Modelling the cloud: Revit is a parametric object oriented software. It lets to create components called families, made by editable and fixed parameters. Once a family is parametric, it is possible to copy it more and more times, only changing the necessary parameters: this feature speed up the whole modelling process.

In this particular case, the "falconatura" was modelled dividing it in three families: in this way it was easier to manage the bonds necessary between the blocks that build this element. Knowing that, inserting in the project three families for each falconatura would distend the modelling time, it was created a nested family that assembled all the three groups: as Pozzoli et al. wrote in 2011, "nesting a family means upload it inside another, in order to create a new one, born with the union of the two."

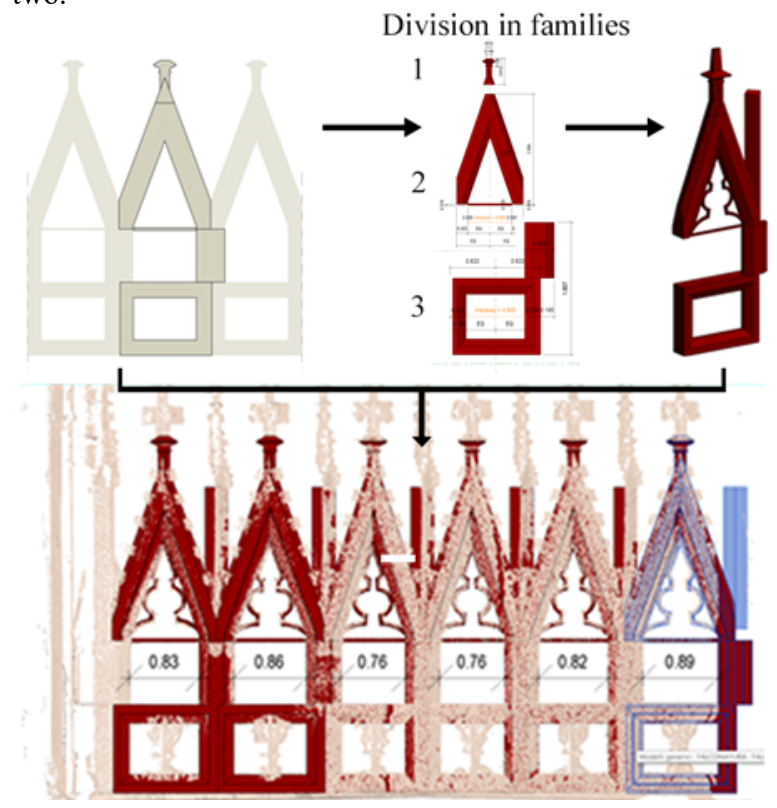

Figure 10. Parametrization workflow in Revit 2016: once modelled one falconatura divided in three families, the others were adjusted locking some parameters and varying others.

The great advantage in using the nested family is that, inserting several copies in the project and editing the interaxle values, all the three parts vary simultaneously. This method works only if, before nesting the three families, it is chosen the same parameter to build them (in this case - generic metric model). In conclusion, once modeled a falconatura in the Families Editor and copying it in the project as a nested family, with the use of dimensions (that can be of various types, e.g. aligned, angular, diameter, radius or arches' length) it is possible to fasten the elements' sizes, or to create the parameters according to how the object has to be modified. In this case the only 
dimensions that change is the inteaxle space between the pillars, while the heights of all the other elements was locked.

Regarding the decorative part, as the elements were unique and complex, first, they were surveyed with photogrammetry and then they were modelled one by one with the help of Rhinoceros and other software that handle the mesh files, and then imported in Revit as SAT file, reducing the number of the mesh's triangles, in order to make the file manageable as it doesn't support too heavy mesh.

\section{CONCLUSIONS}

\subsection{Interoperability tests}

The last step of this research work is to study the interoperability: given that each program has its strength and weaknesses, it is important to have the opportunity to integrate the abilities of the different software. Since Revit has proved to be the most suitable program in the managing and modelling the point cloud, some interoperability tests were run between Rhino and Revit, in order to evaluate the result in terms of maintaining the original shape, possible editing, information attribution and parametrization of the imported model. Before writing the consideration about the interoperability it is important to remember the different way to work of these programs: Rhino employs the NURBS, while the elements exported from Revit are meshes. The difference between these two methods is that the NURBS is a mathematical functions, while meshes are a set of triangular surfaces: this means that the surfaces of the parts created in Revit and exported in Rhino are divided in triangles so they are not homogeneous (consequentially they are also heavier) and vice versa, the NURBS created in Rhino has to be imported in Revit as meshes. To run these tests is interesting to use the IFC (Industry Foundation Classes) canal, as it is the international and recognized format file for open interchange and open BIM. It is commonly used in the interoperability between BIM programs, but recently, a lot of plug-ins improved the use of this format also between BIM and external software - e.g. VisualARQ for Rhino-Revit, Rhino connections for Rhino-Archicad and RhinoGrasshopper-Archicad connection for Grasshopper and Archicad.

\subsection{Connection from Rhino to Revit}

It is possible to import a Rhino file into Revit, only with a SAT format or the IFC one. When it is imported a SAT project in Revit workspace, in this case the falconatura, even if it maintains the shapes, it is not possible to modify it and it is no longer divided in blocks. If it is assigned to a family, it is possible to link all the information and it recognize the volume of the element (this feature is important for computations and the estimation of costs). Regarding the parametrization in the modelling phase, it is not possible to obtain it because, even if the variable constraints are attributed to the elements, it is not possible to modify them, even importing the blocks of the model from Rhino one by one.

On the other hand, with the IFC format - that can be used only with the plug-in VisualARQ for Rhino - the shape and the division in blocks is maintained, but the editing works only with the ones extruded in a linear extrusion and not with the ones extruded through a path. It is also possible to link the information, even if it is not to assign the materials: this is because the blocks are automatically putted in the category "generic models" where it is not possible to assign materials, and without the mean to change it. This issue can be overridden assigning in the plug-in the belonging family, before exporting the model in Revit. The volume is not counted. Regarding the modelling parametrization, it doesn't work because the IFC can't be imported in the families' editor as the SAT format.

\subsection{Connection from Revit to Rhino}

In this case there are much more file format that works from Revit to Rhino, particularly they are: DWG, DXF, OBJ, FBX and IFC. All the format except the last one behave in the same way: they maintain the geometries but not the blocks division (only the three original families), they can partially modify the elements and it is not possible to assign information (even if the volume is recognized) and, above all, it is not possible to turn the mesh into NURBS because, in this particular case, the process fail.

\begin{tabular}{|c|c|c|}
\hline \multicolumn{3}{|c|}{ From Rhino to Revit } \\
\hline & SAT & IFC \\
\hline $\begin{array}{c}\text { Maintaining of } \\
\text { the shapes }\end{array}$ & & \\
\hline $\begin{array}{c}\text { Information } \\
\text { attribution }\end{array}$ & & \\
\hline $\begin{array}{c}\text { Modelling } \\
\text { parametrization }\end{array}$ & & \\
\hline
\end{tabular}

\begin{tabular}{|c|c|c|}
\hline \multicolumn{3}{|c|}{ From Rhino to Revit } \\
\hline $\begin{array}{c}\text { Maintaining of } \\
\text { the shapes }\end{array}$ & SAT & IFC \\
\hline $\begin{array}{c}\text { Information } \\
\text { attribution }\end{array}$ & & \\
\hline $\begin{array}{c}\text { Modelling } \\
\text { parametrization }\end{array}$ & - & \\
\hline
\end{tabular}

Table 5. The performances of the interoperability of the process from Rhino to Revit and vice versa.

\subsection{Final considerations}

\begin{tabular}{|c|c|c|c|c|c|}
\hline & Rhino & Grasshop. & Archicad & Revit \\
\hline \multirow{3}{*}{$\begin{array}{l}\text { Point } \\
\text { cloud }\end{array}$} & Import & 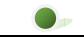 & 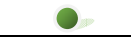 & 0 & - \\
\hline & View & 0 & 0 & o. & - \\
\hline & Editing & 9 & 0 & 0 & 0 \\
\hline \multirow[t]{4}{*}{ Model } & $\begin{array}{c}2 \mathrm{D} \\
\text { retracing }\end{array}$ & - & - & - & 0 \\
\hline & $\begin{array}{c}\text { 3D } \\
\text { modelling }\end{array}$ & - & - & O. & - \\
\hline & Info adding & 0 & 0 & 0 & 0 \\
\hline & $\begin{array}{c}\text { Parametriza } \\
\text { tion }\end{array}$ & - & 0 & 0 & 0 \\
\hline
\end{tabular}

Table 6. Summary of the behaviour of the software regarding the point cloud managing.

This is probably due to the mathematical complexity of this operation, which increases its difficulty depending on the complexity of the element to process. Regarding the IFC files, it doesn't work from Revit to Rhino, and all the attempts of exporting are failed.

Before individuating the right software to obtain a perfect workflow from the point cloud to a BIM system, it is necessary to identify the working method: after the tries made on the same element but with many programs that represent different way to model, the parametric object oriented method is potentially the most suitable, as it is more intuitive than the parametric generative one, and theoretically faster than the direct 
modelling, and between the three typologies, it is the only one that can link information to the modelled elements without the use of external plug-ins.

Still, also in the BIM process, there are some critical issues to be resolved: e.g. as what concern the BIM software Archicad 19 , even if it handles very big point cloud, and it is possible to section it, retracing the profiles and link information, it should implement the modelling part, making it more flexible if the user needs to start the project from the survey data and not from a new project. Considering the evolution that this software has made in the point cloud managing, the issue will be resolved, and the software will become more shaped on the existing building's demand.

At the end of all the tests, nowadays (2016) the most efficient tool to manage the survey data and build a parametric and smart model at 1:50 scale, where is possible to link all the necessary information inside it, is Revit: the software combines the perfect visualization and management of point cloud of Arena 4D with its parametric working method that speed up the modelling phase and lets to insert the technical, historical, restoration, maintenance, etc. data in every single modelled elements.

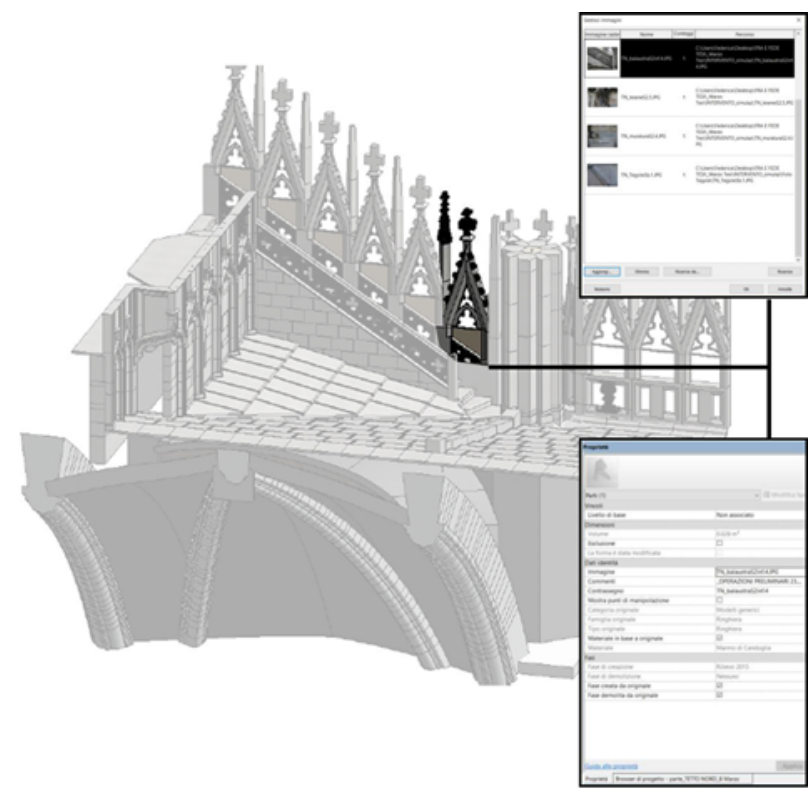

Figure 11. Final parametric model with Revit 16, with the information inside it.

It is necessary to say that as it concern the more complex elements, they still need to be elaborated by other modelling software before importing them in Revit.

Anyway, only in a few years and a few version of the software (precisely from Revit 14) it has evolved, from the need to have a plug-in (Green Spider) to snap the points of the cloud, which had to be imported only in a small size, to the complete management of big survey data. This fact testifies that the concern of these programs is more and more direct towards the existing structures and the Cultural Heritage.

The last observation regards the interoperability between software: analyzing the run tests it is clear that for now the exporting works better from the external software to BIM than vice versa; but, it is also evident that the link that should work better is not consolidated yet. This is not a problem of the connections themselves, but of the approaches to the work that provide that interrelationship. In conclusion, after all the tests is emerged that a single software can't be able to reach all the users' needs, and that the best way to manage and work with Cultural Heritage or the existing buildings and the survey data is to create an approach that combines different methodologies, techniques and software.

\section{ACKNOWLEDGEMENTS}

Strongly thanks to Ing. Francesco Canali, chief of the Veneranda Fabbrica del Duomo Sites, to Francesca Lofurno and Federica Pietrucci for their graduation thesis "BIM e Beni Culturali: un modello informativo per il Duomo di Milano", Arch. Angelo Lagostina for his graduation thesis "Modellazione parametrica real based di oggetti architettonici complessi.", and in general all the colleagues and students that contribute to this work.

\section{REFERENCES}

Achille C., Lombardini N., Tommasi C., 2015. BIM \& Cultural Heritage: compatibility tests on archaeological site. In: Building Information Modelling in Design, Construction and Operations, Bristol, UK, pp. 593-604.

Mandelli A., 2016. Surveying, modeling and management of cultural heritage. Definition of good practices through applications on monuments and artworks. Doctoral thesis, Milano, Italy.

Tedeschi A., 2011. Architettura Parametrica. Introduzione a Grasshopper. Edizioni Le Penseur, Potenza, Italy.

Fassi F., Achille C., Mandelli A., Rechichi F., Parri S., 2015. A new idea of BIM system for visualization, sharing and using huge complex 3D models for facility management. In: The International Archives of the Photogrammetry, Remote Sensing and Spatial Information Sciences, Avila, Spain, Volume XL5/W4.

Rechichi F., Fassi F., Mandelli A., Achille C., 2016. A system for sharing high-resolution models and information on web: the analysis of the web module of bim3dsg system, ISPRS Archives.

Pozzoli S., Villa W., 2010. Revit Architecture 2011. Guida avanzata, Milano, Italy.

Remondino, F. 2011. 3D surveying and modelling of complex architectural sites and heritage objects. In: Disegnarecon, 4, pp. 90-98.

Remondino, F. 2011. Heritage Recording and 3D Modeling with Photogrammetry and 3D Scanning. In: Remote Sens., 3, pp. $1104-1138$ 